\title{
Identification of novel hypermethylated genes and demethylating effect of vincristine in colorectal cancer
}

\author{
Ji Wook Moon', Soo Kyung Lee ${ }^{1}$, Jung Ok Lee ${ }^{1}$, Naml Kim¹, Yong Woo Lee ${ }^{1}$, Su Jin Kim, Ho Jin Kang ${ }^{1}$, Jin Kim², \\ Hyeon Soo Kim ${ }^{1}$ and Sun-Hwa Park ${ }^{1^{*}}$
}

\begin{abstract}
Background: Colorectal cancer (CRC) arises as a consequence of genetic events such as gene mutation and epigenetic alteration. The aim of this study was to identify new hypermethylated candidate genes and methylationbased therapeutic targets using vincristine in CRC.

Methods: We analyzed the methylation status of 27,578 CpG sites spanning more than 14,000 genes in CRC tissues compared with adjacent normal tissues and normal colon tissues using Illumina bead chip array. Twenty-one hypermethylated genes and $18 \mathrm{CpG}$ island methylator phenotype markers were selected as candidate genes. The methylation status of 39 genes was validated by quantitative methylation-specific polymerase chain reaction in CRC tissues, adjacent normal tissues, normal colon cells, and three CRC cell lines. Of these, 29 hypermethylated candidate genes were investigated using the demethylating effects of 5-aza-2'-deoxycytidine (5-aza-dC) and vincristine in CRC cells.
\end{abstract}

Results: Thirty-two out of 39 genes were hypermethylated in CRC tissues compared with adjacent normal tissues. Vincristine induced demethylation of methylated genes in CRC cells to the same extent as 5-aza-dC. The mRNA expression of AKR1B1, CHST10, ELOVL4, FLI1, SOX5, STK33, and ZNF304 was restored by treatment with 5-aza-dC and vincristine.

Conclusion: These results suggest that these novel hypermethylated genes AKR1B1, CHST10, ELOVL4, SOX5, STK33, and ZNF304 may be potential methylation biomarkers and therapeutic targets of vincristine in CRC.

Keywords: Colorectal cancer, Hypermethylated genes, Therapeutic targets, CIMP markers, 5-aza-2'-deoxycytidine, Vincristine

\section{Background}

Colorectal cancer (CRC) is the third most common cancer and the second leading cause of cancer death in the world. CRC is a consequence of genetic events including gene mutations and epigenetic alterations that transform colonic epithelial cells into adenocarcinoma cells [1]. The early detection of CRC is most important in cancer patients to reduce cancer mortality [2]. Different stages of CRC have different prognoses and the effects of adjuvant chemotherapy differ between CRC stage II and

\footnotetext{
* Correspondence: parksh@korea.ac.kr

'Department of Anatomy, Institute of Human Genetics, Korea University College of Medicine, 126-1, Anam-dong 5-ga, Seongbuk-gu, Seoul 136-705, Republic of Korea

Full list of author information is available at the end of the article
}

stage III [3]. Current CRC chemotherapy consists of a combination of cytotoxic DNA antimetabolites, such as 5 -fluorouracil, leucovorin, or oxaliplatin. However, the best combination of these anticancer drugs is still not fully established [4]. To achieve this, epigenetic DNA methylation was reported as a suitable approach for a better understanding of CRC progression and therapeutic targets [5].

A great number of studies have focused on the epigenetic alterations of tumor suppressor genes in the regulation of cancer initiation and progression [6]. Gene-specific methylation changes in promoter $\mathrm{CpG}$ regions have been largely related to biological processes of tumor progression including cell proliferation, communication, adhesion, mobility, signal transduction, and drug resistance [7]. 
Aberrant methylation of CpG islands in the promoter or exon 1 regions of tumor suppressor genes has been correlated with transcriptional silencing of downstream genes in colorectal cancer $[8,9]$. Many genes silenced by aberrant methylation, including CDKN2A, THBS, and SFRP, have been proposed to be associated with $C R C$ tumorigenesis [10-13]. Moreover, promoter methylation was also referred to as the CpG island methylator phenotype (CIMP) [14,15]. CIMP-positive CRC was distinguished from CIMP-negative CRC patients by clinicopathological factors [16], and CIMP was associated with development of the serrated pathway of CRC [17]. Clinically, several CIMPs containing $M L H 1$ (a mismatch repair gene), and microsatellite instability were characterized to be associated with CRC prognosis [18,19]. Furthermore, a panel of CIMP including RUNX3, CACNA1G, IGF2, and MLH1 consists of specific markers for clinical trials [20]. Hughes et al. reviewed the existing literature of 640 potential relevant papers to summarize CIMPs in CRC [21]. Although there are many lines of evidence that have been proposed as potential biomarkers for CRC in humans, many researchers continue to research new CRC-specific methylation markers. Recently, methylation chip array techniques have been widely used to identify new DNA methylation biomarkers in CRC. However, array data are needed to confirm other methods such as quantitative methylation polymerase chain reaction (PCR) (QMSP), methylation-sensitive high-resolution melting, and pyrosequencing [22-24]. QMSP is a sensitive tool and offers quantitative analysis of DNA methylation status [25].

Vincristine is a vinca alkaloid from the plant Catharanthus roseus, and mainly arrests mitosis in metaphase by binding to tubulin dimers [26]. It is used as a chemotherapy drug for various types of cancers, including non-Hodgkin's lymphoma [27], acute lymphoblastic leukemia, lung cancer, breast cancer, and CRC [28-31]. Recently, cyclophosphamide, vincristine, and prednisone (COP) chemotherapy was used to significantly improve overall survival and progression-free survival in primary colonic lymphoma patients [32]. There was one report that low concentration of vincristine reduced the methylated cytosine in human lung adenocarcinoma cells [33]. However, the DNA methylating-based effects of vincristine are still unknown for methylation marker genes in CRC.

In this study, to identify new hypermethylated candidate genes in CRC patients, we analyzed methylation profiles using bead chip array-based technology and QMSP. In addition, to identify methylation-based therapeutic target genes, the demethylating effect of vincristine was examined using 21 hypermethylated candidate genes and 18 CIMP markers. Correlations between methylation status and mRNA expression were analyzed by reverse-transcription PCR.

\section{Methods}

Tissues

Thirty-one pairs of colorectal cancer (CRC) tissues and adjacent normal tissues and 10 normal colon tissues were obtained from the Department of Colorectal Surgery, Korea University Medical Center. The characteristics of each subject are summarized in Table 1. This study was approved by the institutional review board of Korea University and informed consent was obtained (IRB No. KU-IRB-10-08-A-1). The diagnosis of CRC tissues was acquired from pathology reports, the institutional review board, and histological evaluations. Fresh tissue samples were frozen in liquid nitrogen after resection and stored at $-80^{\circ} \mathrm{C}$.

Table 1 Clinicopathologic characteristics of colorectal cancer patients

\begin{tabular}{|c|c|c|c|}
\hline \multirow[t]{2}{*}{ Characteristics } & \multicolumn{2}{|c|}{$\begin{array}{l}\text { Methylation-profiling } \\
\text { subjects }\end{array}$} & \multirow{2}{*}{$\begin{array}{c}\begin{array}{c}\text { Validation } \\
\text { subjects }\end{array} \\
\begin{array}{c}\text { Patients } \\
(n=10)\end{array}\end{array}$} \\
\hline & $\begin{array}{l}\text { Non-patients } \\
(n=10)\end{array}$ & $\begin{array}{l}\text { Patients } \\
(n=21)\end{array}$ & \\
\hline \multicolumn{4}{|l|}{ Age (years) } \\
\hline$>65$ & 4 & 13 & 5 \\
\hline$\leq 65$ & 6 & 8 & 5 \\
\hline \multicolumn{4}{|l|}{ Gender } \\
\hline Male & 2 & 14 & 5 \\
\hline Female & 8 & 7 & 5 \\
\hline \multicolumn{4}{|l|}{ Location } \\
\hline Rectum & & 5 & 5 \\
\hline Right, left colon & 10 & 16 & 5 \\
\hline \multicolumn{4}{|l|}{ Clinical stage } \\
\hline $\mid+\|$ & & 11 & 5 \\
\hline$I I I+I V$ & & 10 & 5 \\
\hline \multicolumn{4}{|l|}{ Differentiation } \\
\hline Well & & 7 & 5 \\
\hline Moderate & & 14 & 5 \\
\hline \multicolumn{4}{|l|}{ Lymphatic metastasis } \\
\hline Present & & 9 & 5 \\
\hline Absent & & 12 & 5 \\
\hline \multicolumn{4}{|c|}{ Maximum tumor diameter (mm) } \\
\hline$>25$ & & 7 & 5 \\
\hline$\leq 25$ & & 14 & 5 \\
\hline \multicolumn{4}{|l|}{ Smoking (1 pack/day) } \\
\hline Yes & & 9 & 4 \\
\hline No & & 12 & 6 \\
\hline \multicolumn{4}{|c|}{ Alcohol intake (3 times/week) } \\
\hline Yes & & 11 & 4 \\
\hline No & & 10 & 6 \\
\hline
\end{tabular}




\section{Cell lines}

One normal colon cell line (CCD18Co) and three CRC cell lines (SW480, Dukes' type B; DLD-1, Dukes' type C; LoVo, Dukes' type $C$ and stage IV) were obtained from the American Type Culture Collection (Manassas, VA, USA). CCD18Co cells were cultured in Eagle's minimum essential medium and the three CRC cells were cultured in RPMI 1640 medium, all supplemented with 10\% fetal bovine serum (Hyclone, Logan, UT, USA) and 1\% penicillin/streptomycin (P/S; Invitrogen), and maintained at $37^{\circ} \mathrm{C}$ and $5 \% \mathrm{CO}_{2}$ atmosphere.

\section{Genomic DNA extraction}

Genomic DNA was extracted using the QIAamp DNA Mini-Kit (Qiagen, Valencia, CA, USA) according to the manufacturer's recommendations. Tissue samples were ground up by 3-mm diameter punches and then mixed with $700 \mu \mathrm{L}$ lysis buffer containing $20 \mu \mathrm{g} / \mathrm{mL}$ Labo Pass protease K (Cosmo Gene Tech., Seoul, Korea), $20 \mathrm{mM}$ Tris. $\mathrm{HCl}$ (pH 8.0), 5 mM EDTA (pH 8.0), $400 \mathrm{mM} \mathrm{NaCl}$, and 1\% SDS solution (Sigma-Aldrich, St. Louis, MO, USA). The mixed samples were incubated at $42^{\circ} \mathrm{C}$ overnight. After incubation, genomic DNA was purified by phenol/chloroform extraction. Genomic DNA was eluted in $100 \mu \mathrm{L}$ of water and quantified with a NanoDrop ND-100 device (Thermo Fisher Scientific, Hudson, $\mathrm{NH}, \mathrm{USA}$ ).

\section{Sodium bisulfite DNA modification}

Two micrograms of genomic DNA in a volume of $20 \mu \mathrm{L}$ RNase-free water was bisulfite-converted using the EpiTect fast DNA bisulfite kit (Qiagen). Bisulfite conversion was performed according to the manufacturer's recommendations. The reaction was performed by mixing $85 \mu \mathrm{L}$ bisulfite mix solution and $35 \mu \mathrm{L}$ DNA protect buffer in $200 \mu \mathrm{L}$ PCR tubes at room temperature. The bisulfite-converted genomic DNA was eluted from the

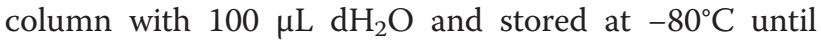
use.

\section{Methylation bead chip array}

Human Methylation 27 DNA Analysis Bead Chip (Illumina Inc., San Diego, CA, USA) is a methylation-profiling technology based on bisulfite modification of DNA. This bead chip array can provide methylation information at a single-base resolution for 27,578 CpG sites spanning more than 14,000 genes. One microgram of bisulfite-converted genomic DNA was applied to the bead chips using Illumina-supplied reagents and conditions. After extension, the array was fluorescently stained and scanned, and the intensities of the $M$ (methylated) and $U$ (unmethylated) bead types were measured. Each methylation data point is represented by fluorescent signals from the $M$ and $\mathrm{U}$ alleles. The ratio of fluorescent signals was then computed from the two alleles; $\beta$ value $=(\max (M, 0)) /$ $(|\mathrm{U}|+|\mathrm{M}|+100)$. The $\beta$ value reflects the methylation level of each CpG site. A $\beta$ value of $0-1.0$ indicates the percent methylation from $0 \%$ to $100 \%$, respectively.

\section{Quantitative methylation-specific PCR (QMSP)}

Quantitative methylation status in the bisulfite-converted genomic DNA was confirmed by quantitative real-time PCR using the 7000 HT Real-Time PCR System (Applied Biosystems) according to the manufacturer's recommendations. Methylation primers for 21 candidate genes and 18 CIMP markers were designed using the MethPrimer software (http://www.urogene.org/methprimer/). Primers for QMSP were designed for large promoter CpG islands containing detected $\mathrm{CpG}$ sites near the transcription start site (Additional file 1: Table S1). PCR reactions were performed using an optical 96-well tray in a final volume of $20 \mu \mathrm{L}$. The reaction mixture consisted of $5 \mu \mathrm{L} 2 \mathrm{X}$ Maxima SYBR Green/ROX qPCR master mix (Thermo Fisher Scientific), $250 \mathrm{nM}$ of each primer, and $30 \mathrm{ng}$ of bisulfiteconverted DNA template. The QMSP program was as follows: $50^{\circ} \mathrm{C}$ for $2 \mathrm{~min}$ and $95^{\circ} \mathrm{C}$ for $10 \mathrm{~min}$, followed by 45 cycles at $95^{\circ} \mathrm{C}$ for $15 \mathrm{~s}$, and then $60^{\circ} \mathrm{C}$ for $1 \mathrm{~min}$. After PCR, a thermal melt profile was performed to examine the homogeneity of the PCR application. Each DNA sample was analyzed in duplicate, and the mean quantity was used for further analysis. Relative quantification of the amplified gene levels in the bisulfite-converted genomic DNA sample was performed by measuring the threshold cycle $\left(C_{\mathrm{T}}\right)$ values of target genes and $\beta$-actin $(A C T B)$. The mean quantity of genes was divided by the mean quantity of $A C T B$ and was used for the normalization of input DNA. The negative values for $A C T B$ were excluded from the methylation analysis. The bisulfite-converted genomic DNA of a known concentration was drawn at 1, 1/4, 1/16, and $1 / 64$ via serial dilutions, and then used in a standard curve for quantification. The modified genomic DNA by CpG methyltransferase M.SssI (NEB, Ipswich, MA, USA) was used as a positive control according to the manufacturer's recommendations. DNA methylation according to M.SssI was verified using the restriction enzyme BstUI (NEB).

\section{Reverse-transcription PCR}

mRNA was extracted using the commercial RNeasy Mini-kit (Qiagen, Hilden, Germany) according to the manufacturer's recommendations. The mRNA was eluted in $20 \mu \mathrm{L}$ of DEPC water (Qiagen) and quantified with a NanoDrop ND-100 device (Thermo Fisher Scientific). One microgram of mRNA from each sample was subjected to cDNA synthesis using Maloney murine leukemia virus RT and random hexamers (Promega, Madison, WI, USA). cDNA synthesis was performed according to the manufacturer's recommendations by mixing $1 \mu \mathrm{L}$ of $1 \mu \mathrm{g}$ 
mRNA, $4 \mu \mathrm{L}$ 5X RT buffer, $1 \mu \mathrm{L} 500 \mathrm{nM}$ oligo dT, $1 \mu \mathrm{L}$ $10 \mathrm{mM}$ dNTP, $0.5 \mu \mathrm{L}$ RNasein, $1 \mu \mathrm{L}$ M-MLV reverse tran-

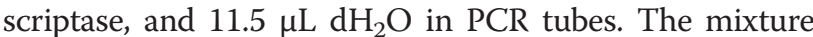
was then incubated at $37^{\circ} \mathrm{C}$ for $1 \mathrm{~h}$. cDNA was diluted

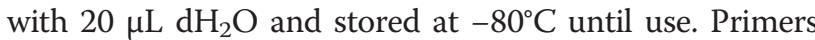
were designed using primer3 version 0.4 .0 (http://primer3. ut.ee/) and are shown in Additional file 1: Table S2. cDNA was amplified by PCR with primers for each target gene. The RT-PCR program was as follows: $95^{\circ} \mathrm{C}$ for $10 \mathrm{~min}$, followed by 35 cycles at $95^{\circ} \mathrm{C}$ for $15 \mathrm{~s}, 60^{\circ} \mathrm{C}$ for $15 \mathrm{~s}$, and then at $72^{\circ} \mathrm{C}$ for $45 \mathrm{~s}$. $A C T B$ was amplified simultaneously with the other PCR products and was used as a control for RNA integrity.

\section{Chemical treatment}

To determine the optimal concentration of 5-aza-2'deoxycytidine (5-aza-dC) and vincristine (Sigma-Aldrich) in CRC cell lines, we measured cell viability with the MTT assay (Additional file 2: Figure S1) according to the manufacturer's recommendations using MTT reagents (10 $\mu \mathrm{L} /$ well, $7.5 \mathrm{mg} / \mathrm{mL}$ in phosphate-buffered saline) and dimethyl sulfoxide (50 $\mu \mathrm{L} /$ well, Sigma-Aldrich). To identify the demethylating effect of treatment with anticancer drugs, CCD18Co, SW480, DLD-1, and LoVo cells were seeded in six-well culture plates (SPL LifeSscience, Pocheon, Korea) at a density of $0.5 \times 10^{5}$ cells per well. After $24 \mathrm{~h}$, cells were cultured in serum-free media containing either $30 \mu \mathrm{M} 5$-aza-dC or $100 \mathrm{nM}$ vincristine in $10 \mu \mathrm{L}$ dimethyl sulfoxide for $48 \mathrm{~h}$ at $37^{\circ} \mathrm{C}$ in a $5 \% \mathrm{CO}_{2}$ atmosphere. After $48 \mathrm{~h}$, cells were washed in PBS (SigmaAldrich) three times and then harvested.

\section{Statistical analysis}

The statistical significance of the methylation bead chip array data was determined using a paired t-test based on $\Delta \beta$ means (means of $\beta$ value in CRC tissues - means of $\beta$ value in normal tissues). The methylated intensity ratio in CRC was confirmed by fold-change (methylated intensity ratio of CRC tissues/methylated intensity ratio of normal colon tissues) and odds ratio (methylated intensity ratio contract of unmethylated intensity in CRC). The false discovery rate (FDR) was controlled by adjusting the $P$ value using the Benjamini-Hochberg algorithm, the incorrectly substituted probabilities of specifically observed methylation CpG sites for each gene in $P$ values. The methylated intensity ratio of QMSP was determined by the percentage of methylated reference (PMR) gene, and the PMR value was defined as $\left[(\mathrm{GENE})_{\text {sample }} /(\mathrm{ACTB})_{\text {sample }}\right] /$ $\left[(\mathrm{GENE})_{\mathrm{M} . S s s} /(\mathrm{ACTB})_{\mathrm{M} . S s s}\right] \times 100$. The significance of different PMR values among CRC tissues and adjacent normal tissues was defined with the chi-squared test and analysis of variance test using Sigma Stat (SPSS Inc., Chicago, IL, USA). All statistical tests were two-sided and $P$ values of $<0.05$ were considered to indicate statistical significance.

\section{Results}

\section{Selection of $\mathbf{2 1}$ hypermethylated candidate genes in CRC}

To identify the aberrant methylation of various genes in CRC, we performed a methylation chip array in 10 normal colon tissues, and 21 CRC tissues and adjacent normal tissues. We found a total of 3,177 CpG sites in the promoter regions (1,704 CpG sites, 53.6\%) and non-promoter

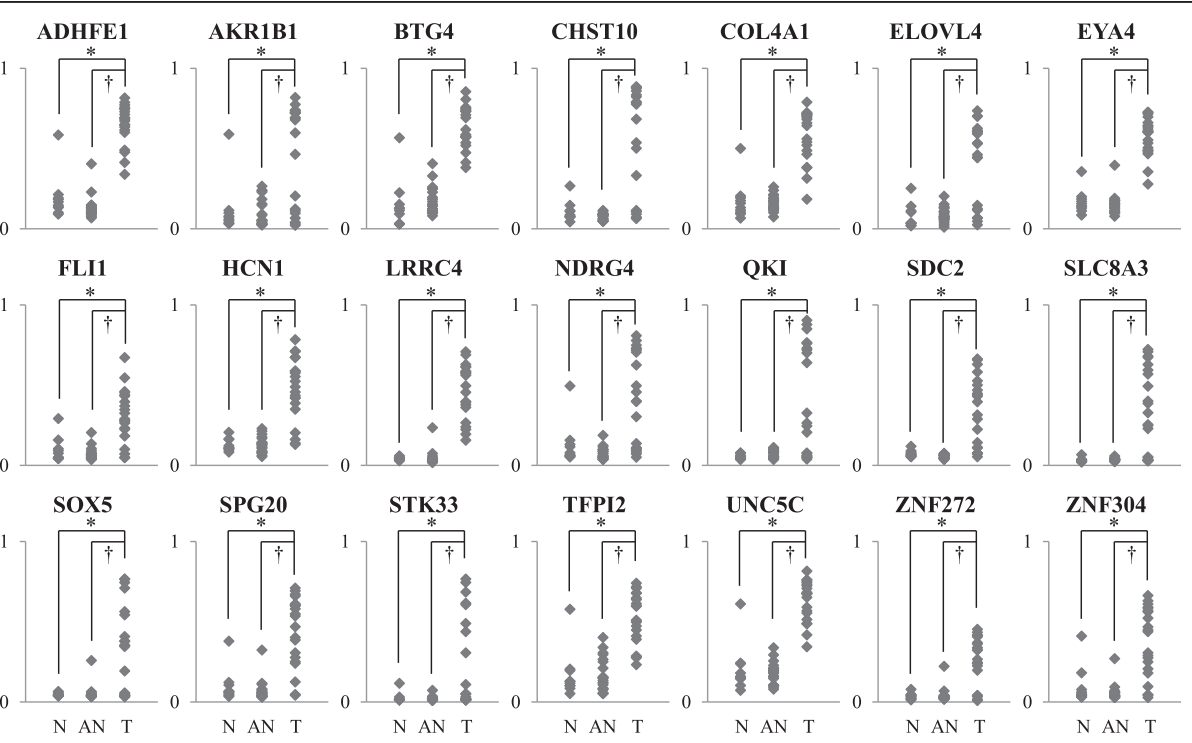

Figure 1 Hypermethylation of promoter CpG sites for $\mathbf{2 1}$ genes by methylation chip array. The methylation statuses of CpG sites in promoter CpG islands for 21 genes were identified using methylation chip array in 10 normal colon tissues, 21 CRC tissues, and 21 adjacent normal tissues. All genes were significantly strongly hypermethylated in their promoter CpG islands in CRC tissues compared with adjacent normal tissues and normal tissues $(P<0.05)$. N: normal colon tissues, T: CRC tissues, AN: adjacent normal tissues. 
regions (1,473 CpG sites, 46.4\%), with aberrant methylated CpG sites identified in CRC tissues compared with adjacent normal and normal colon tissues, according to statistical significance determined by the paired t-test and an FDR $P$ value of $<0.001$ based on a $\Delta \beta$ mean of 0.1 . Among 3,177 CpG sites, we identified 597 genes with hypermethylated CpG sites in promoter CpG islands (data not shown). Finally, we selected 21 candidate genes that contained strongly hypermethylated $\mathrm{CpG}$ sites in promoter CpG islands in CRC tissues compared with adjacent normal tissues (Figure 1).

\section{Validation of 39 genes by QMSP}

To confirm the methylation status of 21 candidate genes from the array results and 18 CIMP markers, we validated the methylation status in the promoter CpG islands of selected genes by QMSP in 10 different CRC tissues compared with adjacent normal tissue. The quantitative analysis with the PMR value supported the differential methylation status between CRC and normal tissues. The methylation status in the promoter $\mathrm{CpG}$ islands of all candidate genes was frequently higher in $\mathrm{CRC}$ tissues compared with adjacent normal tissues except

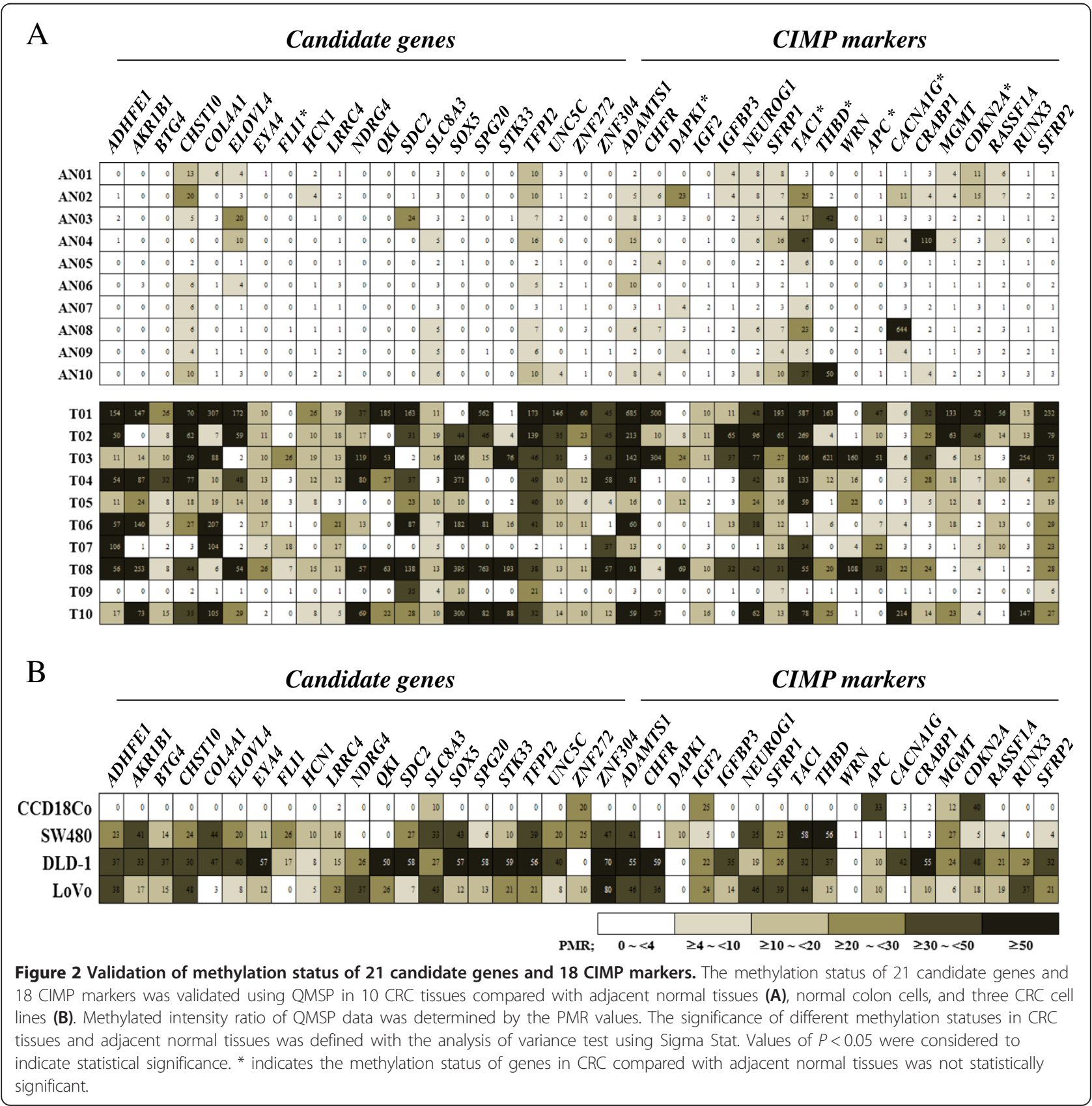


FLI (Figure 2A). The methylation status of 12 CIMP markers, namely, ADAMTS1, CHFR, IGF2, IGFBP3, NEUROG1, SFRP1, WRN, CRABP1, MGMT, RASSF1A, RUNX3, and SFRP2 was also frequently higher in CRC tissues compared with adjacent normal tissues (Figure 2A). In normal colon cells, SLC8A3, ZNF272, IGF2, APC, $M G M T$, and $C D K N 2 A$ were methylated. All genes were hypermethylated in one or more CRC cell lines except WRN (Figure 2B).

\section{Demethylation effect of vincristine on 29}

\section{hypermethylated genes in CRC cell lines}

The 10 genes hypermethylated in normal colon cells or not significantly hypermethylated in tumor tissue were excluded for chemical treatment. Eighteen candidate genes and 11 CIMP markers were selected to identify the demethylating effects of vincristine. The methylation status of 29 genes was determined by PMR values. In normal colon cells, most genes were not affected by 5aza-dC and vincristine treatment (Figure 3A). In contrast, 14 candidate genes and seven CIMP markers were significantly demethylated by 5 -aza-dC treatment in two CRC cell lines (Figure 3B). In addition, 12 candidate genes (ADHFE1, AKR1B1, CHST10, ELOVL4, EYA4, FLI1, QKI, STK33, SOX5, UNC5C, and ZNF304) and eight CIMP markers (ADAMTS1, CHFR, CRABP1, NEUROG1, RUNX3, SFRP1, THBD, and TAC1) were significantly demethylated by vincristine treatment in two more CRC cell lines (Figure 3B).

\section{Restoration of mRNA expression by vincristine in DLD-1} cells

The effect of methylation on mRNA expression was investigated by MSP and RT-PCR analysis in 5-aza-dCand vincristine-treated DLD-1 and CCD18Co cells. The methylation status of CHST10, ELOVL4, EYA4, FLI1,

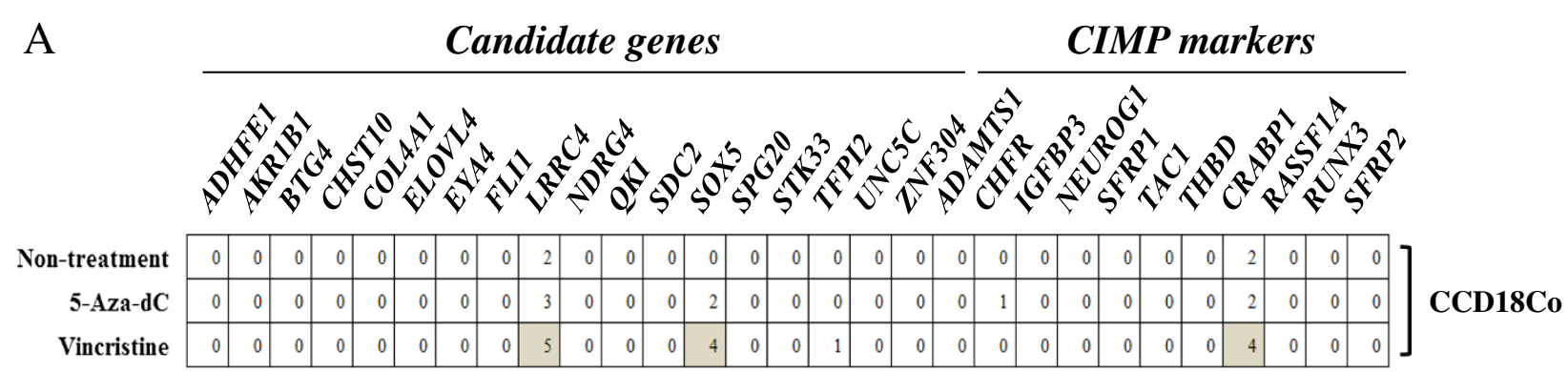

B

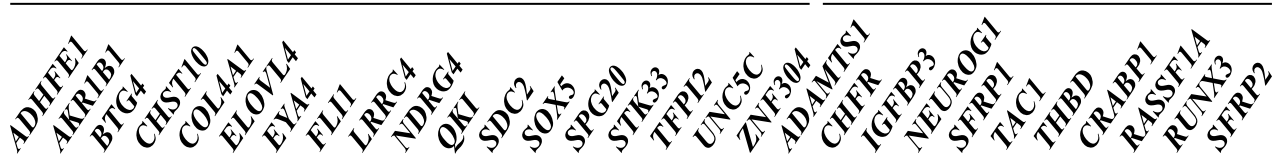

Non-treatment

5-Aza-dC

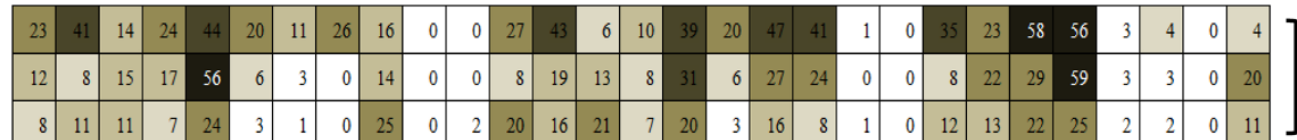

Vincristine

Non-treatment

5-Aza-dC

Vincristine
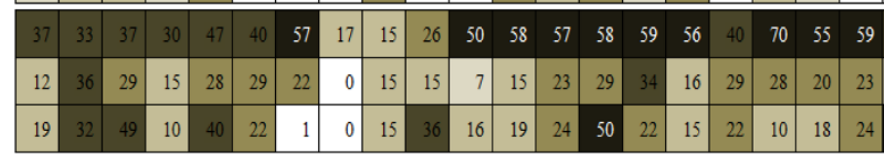

Non-treatment

5-Aza-dC
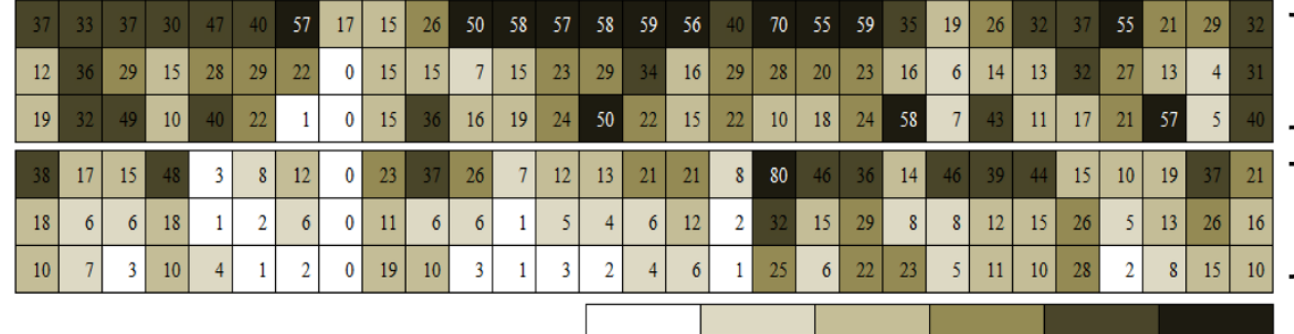

Vincristine

PMIR; $0 \sim<4 \quad \geq 4 \sim<10 \quad \geq 10 \sim<20 \quad \geq 20 \sim<30 \quad \geq 30 \sim<50 \quad \geq 50$

Figure 3 Demethylating effect of 5 -aza-dC and vincristine on methylated genes in three CRC cell lines. Changes in the methylation status of hypermethylated genes in normal colon cells (A) and CRC cells (B) were identified by QMSP after treatment with 30 MM 5 -aza-dC or 100 nM vincristine for $48 \mathrm{~h}$ at $37^{\circ} \mathrm{C}$. Methylated intensity ratio was determined by the percentage of methylated reference (PMR). 
STK33, SOX5, and ZNF304 was decreased by treatment with 5 -aza-dC and vincristine in DLD-1 cells, but were not changed in CCD18Co cells. The methylation status of CHST10, ELOVL4, EYA4, and ZNF304 was highly decreased by vincristine (Figure $4 \mathrm{~A}$ ). The mRNA expression of AKR1B1, CHST10, ELOVL4, FLI1, STK33, SOX5, and ZNF304 was increased by treatment with 5 -aza-dC and vincristine in DLD-1 cells, but EYA4 mRNA expression was not detected. The mRNA expression levels of all genes were not affected by 5 -aza-dC treatment in CCD18Co cells (Figure 4B). The methylation of $A K R 1 B 1$ was not decreased significantly by treatment with 5 -aza$\mathrm{dC}$ or vincristine, but the mRNA expression levels of this gene were increased (Figure 4). These results suggest that vincristine promotes the demethylation of CHST10, ELOVL4, FLI1, SOX5, STK33, and ZNF304, and the methylation-mediated silencing or down expression of these genes was restored by vincristine in DLD-1 cells to the same extent as 5 -aza-dC, as measured by mRNA expression.

\section{Discussion}

This study identified novel hypermethylated genes in CRC through a genome-wide study. DNA hypermethylation leads to the downregulation and silencing of tumor suppressor genes during the pathogenesis of various human cancers [10,34-36]. Recently, genome-wide array-based studies have reported altered DNA methylation gene profiles in CRC [22-24]. Oster et al. identified hypermethylated FLI1, ST6GALNAC5, TWIST1, ADHFE1, JAM2, IRF4, CNRIP1, NRG1, and EYA4 genes in the adenomas and carcinomas of colorectal lesions [22]. Kim et al. also reported 20 top-ranking hypermethylated genes in CRC [23]. Mori et al. identified several novel candidate CRC biomarkers including VSX2, BEND4, NPTX1, BTG4, and GLP1R [24]. In our methylation chip array results, we discovered 1,411 hypermethylation CpG sites that were located in the promoter CpG islands of 597 genes (data not shown), and selected 21 candidate genes for further study (Figure 1). Several candidate genes were consistent with previous reports, such as BTG4, FLI1, TWIST1, ADHFE1, UNC5C, and SPG20 [22-24]. We validated the methylation status in the promoter $\mathrm{CpG}$ islands of candidate genes by QMSP for the investigation of large contiguous CpG sites, the results of which were concordant with the array results for most genes except FLI1 (Figure 2A).

Among the various CIMP markers in CRC, 18 CIMP markers were selected for the validation of methylation status and methylation-based therapeutic targets in CRC. ADAMTS1, CHFR, DAPK1, IGF2, IGFBP3, NEUROG1, SFRP1, TAC1, THBD, and WRN were also hypermethylated in our chip data (data not shown). In our QMSP results of CIMP markers, DAPK1, TAC1, THBD, $A P C, C A C N A 1 G$, and $C D K N 2 A$ were not significantly methylated in CRC tissues (Figure 2A). These discrepancies may be due to differences in the epigenomes of tumors or patient ethnic background.

The hypermethylation of AKR1B1, CHST10, ELOVL4, SOX5, STK33, and ZNF304 have not been previously reported in CRC. $A K R 1 B 1$, aldo-keto reductase family 1, member B1, catalyzes the reduction of aldehydes including the aldehyde form of glucose. It was reported to be downregulated in endometrial cancer and gastric cancer $[37,38]$. The product of CHST10, carbohydrate sulfotransferase 10 ,
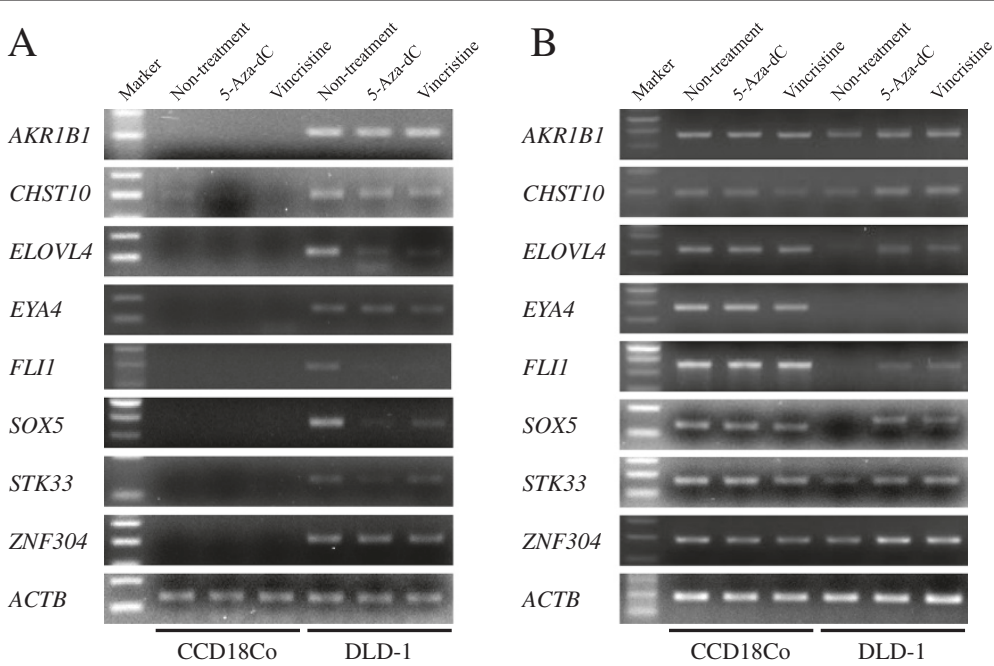

Figure 4 Association between DNA methylation and mRNA expression of eight genes in CCD18Co and DLD-1. The DNA methylation status (A) and mRNA expression (B) of eight genes, including six newly identified hypermethylated genes, was measured using QMSP and RT-PCR in CCD18Co and DLD-1 cells following $30 \mu \mathrm{M}$ 5-aza-dC or $100 \mathrm{nM}$ vincristine treatment for $48 \mathrm{~h}$ at $37^{\circ} \mathrm{C}$. ACTB was amplified simultaneously with the other PCR products and was used as a control for DNA methylation and mRNA integrity. Marker: molecular weight DNA marker. 
is known to inhibit the invasiveness of melanoma cells [39]. ELOVL4 gene product, elongation of very long chain fatty acids 4 , is responsible for the biosynthesis of fatty acids [40]. Hypermethylation of ELOVL4 was reported in hepatocellular carcinoma and pancreatic adenocarcinoma by genome-wide methylation analysis $[41,42]$. SOX5 is a member of the SOX (SRY-related HMG-box) family of transcription factors. It is well known that SOX5 regulates embryonic development and determines cell fate [43]. STK33, serine-threonine kinase 33 , is located on chromosome $11 \mathrm{p} 15.3$, a gene-rich region that has been associated with human diseases and malignancies [44]. ZNF304, zinc finger protein 304, combines two conserved domains, class II AU-rich elements and a Krüppel-associated box, and is associated with the regulation of lymphocyte activation [45].

DNA methylation-mediated silencing of gene expression can be restored by demethylation agents such as 5aza-dC. DNA methyltransferase inhibitor, 5 '-azacytidine, may act as an inducer of cell differentiation by causing de-methylation and re-expression of genes silenced by hypermethylation [46]. 5-Azacytidine was approved in 2004 by the US Food and Drug Administration for treating myelodysplastic syndrome [47], and 5-aza-dC as a 5azacytidine analog was widely used in DNA methylation studies [48]. Vincristine is a microtubule inhibitor and is commonly used for chemotherapy in pediatric acute lymphoblastic patients [32]. Several anticancer drugs are associated with drug-induced DNA hypermethylation in human lung adenocarcinoma and rhabdomyosarcoma cells [33]. Interestingly, the methylated cytosine was reduced after treatment with concentration of vincristine less than $100 \mu$ mole but it was induced after treatment with higher than $1000 \mu$ mole in human lung adenocarcinoma cells [33]. In this study, we selected $100 \mathrm{nM}$ as an optimal concentration of vincristine which does not effect on the viability of CRC cells using MTT assay. Vincristine induced demethylation of methylated genes in CRC cells to the same extent as 5-aza-dC. In addition, vincristine restored the mRNA expression of CHST10, ELOVL4, FLI1, STK33, SOX5, and ZNF304 in CRC cells. Interestingly, the methylation status of $A K R 1 B 1$ was not affected, but its mRNA expression was increased by both drugs. It may be regulated by upstream genes, with a demethylating effect by both drugs. Our results provide insights into the potential functional impact of vincristine on methylated genes in CRC.

\section{Conclusions}

This study has identified novel candidate genes, $A K R 1 B 1$, CHST10, ELOVL4, SOX5, STK33, and ZNF304, and provided evidence for their suitability as methylation biomarkers of CRC. We also analyzed the DNA methylationbased therapeutic effects of vincristine in CRC.

\section{Additional files}

Additional file 1: Table S1. Primers for Quantitative methylation specific PCR (QMSP). Table S2. Primers for mRNA expression.

Additional file 2: Figure S1. Cell viability of CCD18Co, SW480, DLD-1, and LoVo cells by 5-aza-dC and vincristine. Cell viability of four cell lines after treatment with various concentrations of 5-aza-dC and vincristine for three days was evaluated by MTT assay. The cell viability of four cell lines was significantly reduced after treatment with concentration of 5-aza-dC greater than $30 \mu \mathrm{M}$ (A) and did not change significantly after treatment with concentrations of vincristine less than $100 \mathrm{nM}$ for two or three days (B). * $p$-Values of $<0.05$ were considered statistically significant.

\section{Abbreviations}

ACTB: Beta-actin; ADAMTS1: A disintegrin and metalloproteinase with thrombospondin motifs 1; ADHFE1: Alcohol dehydrogenase, iron containing, 1; AKR1B1: Aldo-keto reductase family 1; APC: Antigen-presenting cell; BEND4: BEN domain containing 4; BTG4: B-cell translocation gene 4; CACNA1G: Calcium, voltage-dependent, T type, alpha $1 \mathrm{G}$ subunit; CDKN2A: Cyclin-dependent kinase inhibitor 2A; CHFR: Checkpoint with forkhead and ring finger domains; CHST10: Carbohydrate sulfotransferase 10; CIMP: CpG island methylator phenotype; CNRIP1: Cannabinoid receptor interacting protein 1; COP: Cyclophosphamide, vincristine and prednisone; CRABP1: Cellular retinoic acid-binding protein 1; CRC: Colorectal cancer; DAPK1: Death-associated protein kinase 1; ELOVL4: Elongation of very long chain fatty acids protein 4; EYA4: Eyes absent homolog 4; FDR: False discovery rate; FLI1: Friend leukemia integration 1 transcription factor; GLP1R: Glucagon-like peptide 1 receptor; IGFBP3: Insulin-like growth factorbinding protein 3; IGF2: Insulin-like growth factor 2; IRF4: Interferon regulatory factor 4; JAM2: Junctional adhesion molecule B; MGMT: 0-6methylguanine-DNA methyltransferase; MLH1: MutL homolog 1; M-MLV: Moloney murine leukemia virus; MTT: 3-(4,5-dimethylthiazol-2-yl)-2,5diphenyltetrazolium; NEUROG1: Neurogenin-1; NPTX1: Neuronal pentraxin I; NRG1: Neuregulin 1; QKI: Quaking homolog, KH domain RNA binding; QMSP: Quantitative methylation-specific polymerase chain reaction; PMR: Percentage of methylated reference; RASSF1A: Ras association domaincontaining protein 1; RT-PCR: Reverse transcription polymerase chain reaction; RUNX3: Runt-related transcription factor 3; SLC8A3: Solute carrier family 8, member 3; SFRP1: Secreted frizzled-related protein 1;

SFRP2: Secreted frizzled-related protein 1; SOX5: SPY(sex determining region Y)-box 5; SPG20: Spastic paraplegia 20; STK33: Serine-threonine kinase 33; ST6GALNAC5: ST6 (alpha-N-acetyl-neuraminyl-2,3-beta-galactosyl-1,3)-Nacetylgalactosaminide alpha-2,6-sialyltransferase 5; TAC1: Protachykinin-1; THBD: Thrombomodulin; THBS: Thrombospondin; TWIST1: Twist-related protein 1; UNC5C: Unc-5 homolog C; VSX2: Visual system homeobox 2; WRN: Werner syndrome; ZNF272: Zinc finger protein 272; ZNF304: Zinc finger protein 304; 5-aza-dC: 5-aza-2'-deoxycytidine.

\section{Competing interests}

The authors declare that they have no competing interests.

\section{Authors' contributions}

JWM designed the study and drafted the manuscript. SKL and YWL prepared the clinical specimens and participated in the organization of clinical data. Dr. JOL, NK and SJK participated in the analysis of methylation array results and searching references. Dr. HJK helped the arrangement of results. Prof. JK provided the clinical specimens and clinicopathologic informations. Prof. HSK helped analyzed the results and revised the manuscript. Prof. SHP supervised the study and revised the manuscript. All authors read and approved the final manuscript.

\section{Acknowledgements}

This study was supported by the Basic Science Research Program through the National Research Foundation of Korea (NRF) funded by the Ministry of Education, Science and Technology (grant number: 2010-0024117).

\section{Author details}

'Department of Anatomy, Institute of Human Genetics, Korea University College of Medicine, 126-1, Anam-dong 5-ga, Seongbuk-gu, Seoul 136-705, Republic of Korea. ${ }^{2}$ Department of General Surgery, Korea University Medical Center, 126-1, Anam-dong 5-ga, Seongbuk-gu, Seoul 136-705, Republic of Korea. 
Received: 4 December 2013 Accepted: 2 January 2014

Published: 6 January 2014

\section{References}

1. Grady WM, Carethers JM: Genomic and epigenetic instability in colorectal cancer pathogenesis. Gastroenterology 2008, 135:1079-1099.

2. Kim MS, Lee J, Sidransky D: DNA methylation markers in colorectal cancer. Cancer Metastasis Rev 2010, 29:181-206.

3. Dotan $\mathrm{E}$, Cohen SJ: Challenges in the management of stage II colon cancer. Semin Oncol 2011, 38:511-520.

4. Alberts SR, Sargent DJ, Nair S, Mahoney MR, Mooney M, Thibodeau SN, Smyrk TC, Sinicrope FA, Chan E, Gill S, et al: Effect of oxaliplatin, fluorouracil, and leucovorin with or without cetuximab on survival among patients with resected stage III colon cancer: a randomized trial. JAMA 2012, 307:1383-1393.

5. Vogelstein B, Fearon ER, Hamilton SR, Kern SE, Preisinger AC, Leppert M, Nakamura Y, White R, Smits AM, Bos JL: Genetic alterations during colorectal-tumor development. N Engl J Med 1988, 319:525-532.

6. Lao W, Grady WM: Epigenetics and colorectal cancer. Nat Rev Gastroenterol Hepatol 2011, 8:686-700.

7. Pogribny IP, Beland FA: DNA methylome alterations in chemical carcinogenesis. Cancer Lett 2013, 334:39-45.

8. Kondo Y, Issa JP: Epigenetic changes in colorectal cancer. Cancer Metastasis Rev 2004, 23:29-39.

9. Hinoue $T$, Weisenberger DJ, Lange $C P$, Shen $H$, Byun HM, Van Den Berg D, Malik S, Pan F, Noushmehr H, van Dijk CM, et al: Genome-scale analysis of aberrant DNA methylation in colorectal cancer. Genome Res 2012, 22:271-282

10. Baylin SB, Herman JG: DNA hypermethylation in tumorigenesis: epigenetics joins genetics. Trends Genet 2000, 16:168-174.

11. Rashid A, Shen L, Morris JS, Issa JP, Hamilton SR: CpG island methylation in colorectal adenomas. Am J Pathol 2001, 159:1129-1135.

12. Suzuki H, Watkins DN, Jair KW, Schuebel KE, Markowitz SD, Chen WD, Pretlow TP, Yang B, Akiyama Y, Van Engeland M, et al: Epigenetic inactivation of SFRP genes allows constitutive WNT signaling in colorectal cancer. Nat Genet 2004, 36:417-422.

13. Rodriguez J, Frigola J, Vendrell E, Risques RA, Fraga MF, Morales C, Moreno V, Esteller M, Capella G, Ribas M, Peinado MA: Chromosomal instability correlates with genome-wide DNA demethylation in human primary colorectal cancers. Cancer Res 2006, 66:8462-9468.

14. Weisenberger DJ, Siegmund KD, Campan M, Young J, Long TI, Faasse MA, Kang GH, Widschwendter M, Weener D, Buchanan D, et al: CpG island methylator phenotype underlies sporadic microsatellite instability and is tightly associated with BRAF mutation in colorectal cancer. Nat Genet 2006, 38:787-793.

15. Ogino S, Cantor M, Kawasaki T, Brahmandam M, Kirkner GJ, Weisenberger DJ, Campan M, Laird PW, Loda M, Fuchs CS: CpG island methylator phenotype (CIMP) of colorectal cancer is best characterised by quantitative DNA methylation analysis and prospective cohort studies. Gut 2006, 55:1000-1006.

16. Noffsinger AE: Serrated polyps and colorectal cancer: new pathway to malignancy. Annu Rev Pathol 2009, 4:343-364.

17. Snover DC: Update on the serrated pathway to colorectal carcinoma. Hum Pathol 2011, 42:1-10.

18. Dahlin AM, Palmqvist R, Henriksson ML, Jacobsson M, Eklof V, Rutegard J, Oberg A, Van Guelpen BR: The role of the CpG island methylator phenotype in colorectal cancer prognosis depends on microsatellite instability screening status. Clin Cancer Res 2010, 16:1845-1855.

19. Ogino S, Nosho K, Kirkner GJ, Kawasaki T, Meyerhardt JA, Loda M, Giovannucci EL, Fuchs CS: CpG island methylator phenotype, microsatellite instability, BRAF mutation and clinical outcome in colon cancer. Gut 2009, 58:90-96.

20. Ogino S, Kawasaki T, Kirkner GJ, Kraft P, Loda M, Fuchs CS: Evaluation of markers for $\mathrm{CpG}$ island methylator phenotype (CIMP) in colorectal cancer by a large population-based sample. J Mol Diagn 2007, 9:305-314

21. Hughes LA, Khalid-de Bakker CA, Smits KM, Van den Brandt PA, Jonkers D, Ahuja N, Herman JG, Weijenberg MP, Van Engeland M: The CpG island methylator phenotype in colorectal cancer: progress and problems. Biochim Biophys Acta 2012, 1825:77-85.

22. Oster B, Thorsen K, Lamy P, Wojdacz TK, Hansen LL, Birkenkamp-Demtroder K, Sorensen KD, Laurberg S, Orntoft TF, Andersen CL: Identification and validation of highly frequent $\mathrm{CpG}$ island hypermethylation in colorectal adenomas and carcinomas. Int J Cancer 2011, 129:2855-2866.

23. Kim YH, Lee HC, Kim SY, Yeom Yl, Ryu KJ, Min BH, Kim DH, Son HJ, Rhee PL, Kim JJ, et al: Epigenomic analysis of aberrantly methylated genes in colorectal cancer identifies genes commonly affected by epigenetic alterations. Ann Surg Oncol 2011, 18:2338-2347.

24. Mori Y, Olaru AV, Cheng Y, Agarwal R, Yang J, Luvsanjav D, Yu W, Selaru FM, Hutfless S, Lazarev M, et al: Novel candidate colorectal cancer biomarkers identified by methylation microarray-based scanning. Endocr Relat Cancer 2011, 18:465-478.

25. Eads CA, Danenberg KD, Kawakami K, Saltz LB, Blake C, Shibata D, Danenberg PV, Laird PW: MethyLight: a high-throughput assay to measure DNA methylation. Nucleic Acids Res 2000, 28:E32.

26. Tobin WE, Sandler G: Depression of muscle spindle function with vincristine. Nature 1966, 212:90-91.

27. Yahalom J, Varsos G, Fuks Z, Myers J, Clarkson BD, Straus DJ: Adjuvant cyclophosphamide, doxorubicin, vincristine, and prednisone chemotherapy after radiation therapy in stage I low-grade and intermediate-grade non-Hodgkin lymphoma. Results of a prospective randomized study. Cancer 1993, 71:2342-2350.

28. Gingrich RD, Armitage JO, Burns CP: Treatment of adult acute lymphoblastic leukemia with cytosine arabinoside, vincristine, and prednisone. Cancer Treat Rep 1978, 62:1389-1391.

29. Kolarić K, Roth A, Vukas D: Combination chemotherapy with adriamycin, cyclophosphamide, methotrexate and vincristine in lung cancer patients with extensive disease. Tumori 1979, 65:635-642.

30. Taylor CW, Dalton WS, Mosley K, Dorr RT, Salmon SE: Combination chemotherapy with cyclophosphamide, vincristine, adriamycin, and dexamethasone (CVAD) plus oral quinine and verapamil in patients with advanced breast cancer. Breast Cancer Res Treat 1997, 42:7-14.

31. Fleischer I, Wainstein R, de Gibson AS: Treatment of advanced cancer of the colon and rectum with the combination of 5 -fluorouracil, imidazolecarboxamide and vincristine. Medicina (B Aires) 1983, 43:143-146.

32. Tang TC, Kuo MC, Chang H, Dunn P, Wang PN, Wu JH, Lin TL, Hung YS, Kuo $\Pi$, Shih LY: Primary colonic lymphoma: an analysis of 74 cases with localized large-cell lymphoma. Eur J Haematol 2011, 87:28-36.

33. Nyce J: Drug-induced DNA hypermethylation and drug resistance in human tumors. Can Res 1989, 49:5829-5836.

34. Maruyama R, Sugio K, Yoshino I, Maehara Y, Gazdar AF: Hypermethylation of FHIT as a prognostic marker in nonsmall cell lung carcinoma. Cancer 2004, 100:1472-1477.

35. Uhm KO, Lee ES, Lee YM, Kim HS, Park YN, Park SH: Aberrant promoter CpG islands methylation of tumor suppressor genes in cholangiocarcinoma. Oncol Res 2008, 17:151-157.

36. Uhm KO, Lee JO, Lee YM, Lee ES, Kim HS, Park SH: Aberrant DNA methylation of integrin alpha4: a potential novel role for metastasis of cholangiocarcinoma. J Cancer Res Clin Oncol 2010, 136:187-194.

37. Hevir N, Sinkovec J, Lanisnik Rizner T: Decreased levels of AKR1B1 and AKR1B10 in cancerous endometrium compared to adjacent noncancerous tissue. Chem Biol Interact 2013, 202:226-233.

38. Kropotova ES, Zinov'eva OL, Zyrianova AF, Choinzonov EL, Afanas'ev SG, Cherdyntseva NV, Beresten SF, Oparina N, Mashkova TD: Expression of genes involved in retinoic acid biosynthesis in human gastric cancer. Mol Biol (Mosk) 2013, 47:317-330.

39. Zhao X, Graves C, Ames SJ, Fisher DE, Spanjaard RA: Mechanism of regulation and suppression of melanoma invasiveness by novel retinoic acid receptor-gamma target gene carbohydrate sulfotransferase 10 . Can Res 2009, 69:5218-5225.

40. Zhang K, Kniazeva M, Han M, Li W, Yu Z, Yang Z, Li Y, Metzker ML, Allikmets R, Zack DJ, et al: A 5-bp deletion in ELOVL4 is associated with two related forms of autosomal dominant macular dystrophy. Nat Genet 2001 27:89-93.

41. Revill K, Wang T, Lachenmayer A, Kojima K, Harrington A, Li J, Hoshida Y, Llovet JM, Powers S: Genome-Wide Methylation Analysis and Epigenetic Unmasking Identify Tumor Suppressor Genes in Hepatocellular Carcinoma. Gastroenterology 2013, 145:1424-1435.

42. Omura N, Li CP, Li A, Hong SM, Walter K, Jimeno A, Hidalgo M, Goggins M: Genome-wide profiling of methylated promoters in pancreatic adenocarcinoma. Cancer Biol Ther 2008, 7:1146-1156.

43. Lefebvre $V$ : The SoxD transcription factors-Sox5, Sox6, and Sox13-are key cell fate modulators. Int J Biochem Cell Biol 2010, 42:429-432. 
44. Nowak NJ, Shows TB: Genetics of chromosome 11: loci for pediatric and adult malignancies, developmental disorders, and other diseases.

Cancer Invest 1995, 13:646-659.

45. Sabater L, Ashhab Y, Caro P, Kolkowski EC, Pujol-Borrell R, Dominguez O: Identification of a KRAB-containing zinc finger protein, ZNF304, by AUmotif-directed display method and initial characterization in lymphocyte activation. Biochem Biophys Res Commun 2002, 293:1066-1072.

46. Kaminskas E, Farrell AT, Wang YC, Sridhara R, Pazdur R: FDA drug approval summary: azacitidine (5-azacytidine, Vidaza) for injectable suspension. Oncologist 2005, 10:176-182.

47. Peedicayil J: Epigenetic therapy-a new development in pharmacology Indian J Med Res 2006, 123:17-24.

48. Christman JK: 5-Azacytidine and 5-aza-2'-deoxycytidine as inhibitors of DNA methylation: mechanistic studies and their implications for cancer therapy. Oncogene 2002, 21:5483-5495.

doi:10.1186/1756-9966-33-4

Cite this article as: Moon et al:: Identification of novel hypermethylated genes and demethylating effect of vincristine in colorectal cancer.

Journal of Experimental \& Clinical Cancer Research 2014 33:4.

\section{Submit your next manuscript to BioMed Central and take full advantage of:}

- Convenient online submission

- Thorough peer review

- No space constraints or color figure charges

- Immediate publication on acceptance

- Inclusion in PubMed, CAS, Scopus and Google Scholar

- Research which is freely available for redistribution 